\title{
The Feasibility of 3D Printing Technology on the Treatment of Pilon Fracture and Its Effect on Doctor-Patient Communication
}

\author{
Wenhao Zheng, Chunhui Chen, Chuanxu Zhang, Zhenyu Tao, and Leyi Cai $\mathbb{D}$ \\ Department of Orthopaedic Surgery, The Second Affiliated Hospital and Yuying Children's Hospital of Wenzhou Medical University, \\ Wenzhou 325000, China
}

Correspondence should be addressed to Leyi Cai; caileyi1990@163.com

Received 20 September 2017; Accepted 19 December 2017; Published 18 January 2018

Academic Editor: Frankie Leung

Copyright (C) 2018 Wenhao Zheng et al. This is an open access article distributed under the Creative Commons Attribution License, which permits unrestricted use, distribution, and reproduction in any medium, provided the original work is properly cited.

\begin{abstract}
Purpose. The aim of this study was to assess the feasibility and effectiveness of the three-dimensional (3D) printing technology in the treatment of Pilon fractures. Methods. 100 patients with Pilon fractures from March 2013 to December 2016 were enrolled in our study. They were divided randomly into 3D printing group $(n=50)$ and conventional group $(n=50)$. The 3D models were used to simulate the surgery and carry out the surgery according to plan in $3 \mathrm{D}$ printing group. Operation time, blood loss, fluoroscopy times, fracture union time, and fracture reduction as well as functional outcomes including VAS and AOFAS score and complications were recorded. To examine the feasibility of this approach, we invited surgeons and patients to complete questionnaires. Results. 3D printing group showed significantly shorter operation time, less blood loss volume and fluoroscopy times, higher rate of anatomic reduction and rate of excellent and good outcome than conventional group $(P<0.001, P<0.001, P<0.001, P=0.040$, and $P=0.029$, resp.). However, no significant difference was observed in complications between the two groups $(P=0.510)$. Furthermore, the questionnaire suggested that both surgeons and patients got high scores of overall satisfaction with the use of 3D printing models. Conclusion. Our study indicated that the use of 3D printing technology to treat Pilon fractures in clinical practice is feasible.
\end{abstract}

\section{Introduction}

Tibia Pilon fractures are complex injuries constituting $1 \%$ of all lower-extremity fractures and $5 \%$ to $10 \%$ of tibia fractures [1]. These fractures often result from high-energy trauma leading to multiple metaphyseal fragments, bone loss, displaced intra-articular comminution, and severe soft tissue injuries, which makes them one of the most difficult fractures to treat [2]. The fibula is usually fractured in these highenergy injuries. However, the treatment of Pilon fractures is a matter of controversy. The aim of operative treatment is anatomic reduction of the articular fragments and restoration of the distal tibial alignment while avoiding additional soft tissue trauma [3]. Most surgeons have recommended immediate open reduction and internal fixation (ORIF) after injury, because it can achieve anatomical reconstruction of the articular surface and satisfying functional outcomes postoperatively while sparing the soft tissue $[4,5]$. Nevertheless, various complications, including soft tissue problems, delayed union, nonunion, malunion, implant failure, joint stiffness, and posttraumatic arthritis, may easily arise after surgery if not treated properly, which results in severe pain and affects the motor functions of the patients $[6,7]$.

In order to achieve better outcomes, we need more clinical exploration. ORIF need to be more accurate and individualized. Three-dimensional (3D) printing is a rapid prototyping technology that uses a 3D digital model to physically build an object in layer. In recent years, the use of $3 \mathrm{D}$ printing has allowed for the rapid manufacturing of custom-designed implants for orthopedics and reconstructive surgery, which can assist in accurate preoperative planning, as well as surgical strategy simulation and enhance communication with patients [8-10].

In this study, we compared the traditional surgery with surgery assisted by $3 \mathrm{D}$ printing technology in the treatment of Pilon fractures and ifs feasibility, efficacy, and safety will be included. Moreover, the communicative effectiveness of $3 \mathrm{D}$ printing between doctors and patients will also be investigated. 


\section{Materials and Methods}

2.1. Patients. From March 2013 to December 2016, 100 patients with Pilon fractures were enrolled in this study. The inclusion criteria were as follows: (1) age older than 18 years old; (1) fresh closed fractures (within two weeks from injury), (2) unilateral Pilon fractures, (3) the contralateral normal tibia should not have any fracture, deformity, or history of surgery, and (4) at least 12 months of follow-up. The exclusion criteria were as follows: (1) the contralateral tibia fractures and/or dislocation, (2) old and pathological fractures, (3) open fractures, (4) severe soft tissue injuries (AO closed soft tissue injury grades [11] IC4 and IC5), and (5) multiple fractures.

The fourth author of this study, who was not involved in clinical treatment, was responsible for assigning the patients to the two groups and subsequent data collection and statistics analysis. The 100 patients were randomized to conventional group (50 cases) and 3D printing group (50 cases) by random number table method. The flowchart of the study patients is presented in Figure 1. All the operations were performed by the same team. This study was approved by the Institutional Review Board of The Second Affiliated Hospital of Wenzhou Medical University.

2.2. Printing the 3D Model. We received CT scans of Pilon fracture patients from the Star PACS system (INFINITT, Seoul, South Korea) of our hospital. The original CT data were stored in DICOM format and 3-dimensional (3D) reconstructed using Mimics software v17.0 (Materialise, Leuven, Belgium), positioning by adjusting the threshold to reveal the intact structures of tibia and the bones around the ankle joint. The Region Growing command was used to separate the bones and soft tissues and establish the Mask of the tibia. The pixel set of the tibia was processed using the Calculate 3D form Mask command to produce the mirror image of the contralateral side, which was used as the 3D model of the injured side. The Mask pixel set of each fragment was established and the 3D Object was calculated using the Mask. The 3D model of the injured tibia was produced using Unite Boolean calculation and further processing for the noise reduction and smoothing of the tibia. The design data was then imported date into the $3 \mathrm{D}$ printing software (Cura Software v15.02) in STL format. After a 3D digital model was formed, we saved it in Gcode format and exported it to a 3D printer (3D ORTHO Waston Med, Inc., Changzhou, Jiangsu, China). Finally, the exact $1: 1$ models of the injured tibia and the mirrored contralateral tibia were fabricated.

2.3. Surgery Simulation. Surgeons were able to simulate the operation in vitro though the fracture model and the mirror model of normal side tibia. The structural feature of fracture was clear in the $3 \mathrm{D}$ printing models, and surgeons could mimic the intraoperative reduction and fixation maneuver accurately on the models. Moreover, we could choose suitable metal plates and screws in the real-size tibia model. The ideal length, location, and orientation were placed on the model.
Then, the X-ray of the model would be taken for checking the proper position of the plate and the screws, which would be sterilized and stored for later use in surgery. In addition, the $3 \mathrm{D}$ printed model can be used intraoperatively as a reference for anatomical reduction of the fracture.

2.4. Surgical Methods. All the surgical procedure was performed by the same team as described previously [11]. The patients were subject to epidural anesthesia at the affected site and placed into the supine position on a standard radiolucent bed. Pneumatic tourniquet was applied to occlude the blood circulation. A broader approach to achieve satisfactory reduction was used as needed according to the fracture pattern. The skin and subcutaneous tissues were opened layer by layer. The tibial Pilon fracture was exposed clearly and the periosteum was appropriately stripped. After the elimination of soft tissues on the fracture bone, anatomical reduction of the fracture was made under direct vision. Before reduction, sufficient traction was used to maintain the reduction, and the obtained image was compared with radiographs of the contralateral ankle to ensure the reconstruction of the distal tibiofibular surface. The fracture fragments were fixed with Kirschner wires temporarily after the reduction. In 3D printing group, the preselected and prefabricated plate and screws determined by the 3D-printed model simulation were placed to fix fracture. However, in conventional group, the selection of plate and screws were only determined by the measurement during the surgery. The reduction was evaluated by the intraoperative C-arm X-ray machine. Finally, the incision was closed as usual.

2.5. Postoperative Management. The postoperative management was the same for all patients. The antibiotics were used within 3 days postoperatively. After operation, pressure dressing was applied on the wound surface and the affected side was raised to relieve the swelling around the wound. A postoperative X-ray of the affected tibia was taken the day after surgery. All patients remained strict toe-touch weightbearing immediately postoperatively for approximately 3 months. A graduated physical therapy protocol was initiated on postoperative day 1 with crutches or a walker. The protocol was directed at gait training, active and passive lower-extremity range of motion exercises, and endurance training. Cast immobilization was used until wound healing, followed by immobilization with a removable cast-type boot. At approximately 3 months, weight-bearing was advanced as tolerated with supervision of physical therapy. This was followed by weaning of assistive devices for gait and the initiation of proprioception and endurance activities. The functional outcome of ankle was evaluated by the American Orthopaedic Foot and Ankle Society (AOFAS) hindfoot scores and visual analog scale (VAS) pain scores. The VAS pain score was used to measure the amount of pain patients felt between 0 and 10 points and contained word descriptors. The 100-point AOFAS scoring system considers a score of $\geq 90$ points as excellent, $80-89$ points as good, and $70-79$ points as fair and a score of $\leq 69$ points as poor [12]. 


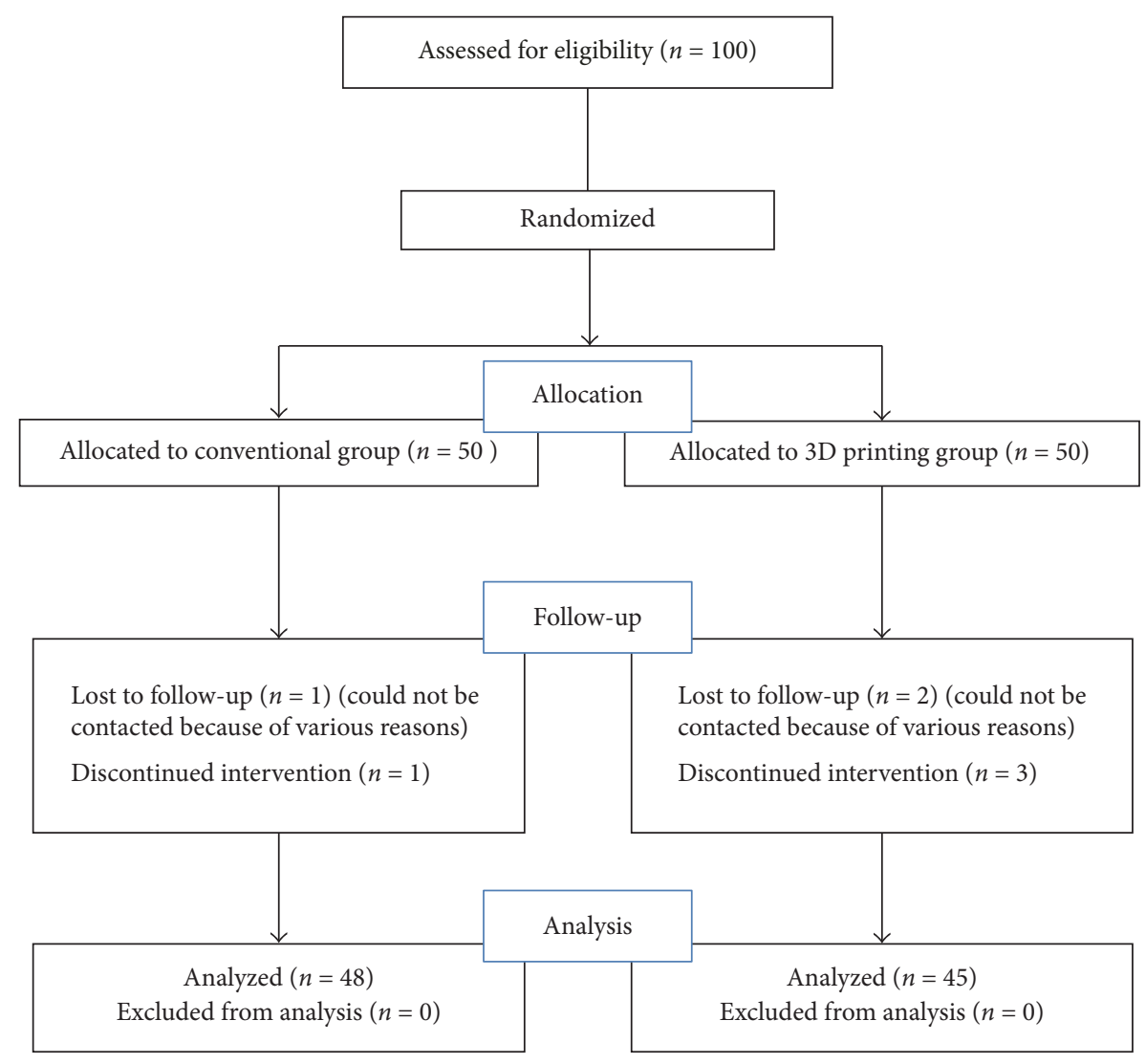

FIgURE 1: The flowchart of the study patients.

2.6. Assessment Parameters. The operation time, blood loss volume, times of fluoroscopy during the surgery, and fracture union time were recorded. The fracture reduction was assessed on orthogonal simple radiographs and graded according to the score described by Burwell and Charnley [13]. The functional outcomes of ankle were evaluated by the range of ankle motion (dorsiflexion and plantarflexion), AOFAS, and VAS scores. Furthermore, the complications of the two groups were also evaluated in our study. The fracture healing was assessed radiographically through callus formation. We considered a healing time of less than 6 months as normal, between 6 and 9 months as a delayed union, and more than 9 months as nonunion [14]. Malunion was defined as more than 5 degrees of angular or rotational deformity [15]. Posttraumatic arthritis was described as painful range of motion with radiographic evidence of a narrowed joint space.

2.7. Questionnaire. A questionnaire was designed to allow doctors to evaluate the models. The details of the questionnaire were shown in Table 4. Ten orthopaedic surgeons evaluated the verisimilitude and effectiveness of the life-sized $3 \mathrm{D}$ model using the questionnaire. Besides, a questionnaire was also designed for patients and nonmedical professionals. This questionnaire was used to assess patient satisfaction with the levels of preoperative communication between the two groups. The details of the questionnaire were shown in Table 5. The scores ranged from 1 to 10 points, 1 point indicated that the model was useless/very poor/not realistic at all, and 10 points indicated that the model was very useful/very good/very realistic.

2.8. Statistical Analysis. Data were analyzed using Student's $t$ unpaired test and the chi-squared test. $P<0.05$ was considered statistically significant. Data are given as mean \pm standard deviation. Statistical analyses were performed using SPSS version 18.0 software.

\section{Results}

3.1. Patient Characteristics. Of the admitted patients, a total of 7 patients ( 2 patients in conventional group and 5 patients in $3 \mathrm{D}$ printing group) were lost to follow-up because of various reasons. As a result, there were 48 patients remaining in the conventional group and 45 patients in the $3 \mathrm{D}$ printing group. There were 31 males and 17 females in conventional group and 35 males and 10 females in $3 \mathrm{D}$ printing group. Average age for patients in conventional group was $42.5 \pm$ 9.0 years and in $3 \mathrm{D}$ printing group was $41.2 \pm 9.3$ years. In conventional group, the fracture was on the right in 28 patients and the left in 20 . In 3D printing group, the fracture was on the right in 22 patients and the left in 23. According to the AO fracture classification [16], conventional group had 8 patients with type 43-C1 fracture, 17 patients with type 43-C2 fracture, and 23 patients with type $43-\mathrm{C} 3$ fracture, whereas 
TABLE 1: Comparison of clinical data between the two groups.

\begin{tabular}{|c|c|c|c|c|}
\hline & Conventional group $(n=48)$ & $3 \mathrm{D}$ printing group $(n=45)$ & $t$ or $\chi^{2}$ & $P$ value \\
\hline Operation time (min) & $90.2 \pm 10.9$ & $74.1 \pm 8.2$ & 8.021 & $<0.001$ \\
\hline Blood loss volume (ml) & $159.8 \pm 26.5$ & $117.1 \pm 20.7$ & 8.625 & $<0.001$ \\
\hline Times of fluoroscopy $(n)$ & $11.0 \pm 2.9$ & $7.6 \pm 2.2$ & 6.328 & $<0.001$ \\
\hline Fracture union time (month) & $5.3 \pm 1.2$ & $5.0 \pm 1.1$ & 1.013 & 0.314 \\
\hline Fracture reduction & - & - & 4.305 & 0.116 \\
\hline Anatomic $(n)$ & 36 & 41 & 4.232 & 0.040 \\
\hline Fair $(n)$ & 8 & 3 & 2.227 & 0.136 \\
\hline Poor $(n)$ & 4 & 1 & 0.715 & $0.398^{\mathrm{a}}$ \\
\hline Rate of anatomic reduction (\%) & $75 \%$ & $91.1 \%$ & 4.232 & 0.040 \\
\hline
\end{tabular}

${ }^{\mathrm{a} P} \mathrm{v}$ value for continuity-corrected chi-squared test.

TABLE 2: Comparison of functional outcomes between the two groups.

\begin{tabular}{|c|c|c|c|c|}
\hline & Conventional group $(n=48)$ & $3 \mathrm{D}$ printing group $(n=45)$ & $t$ or $\chi^{2}$ & $P$ value \\
\hline Follow-up time (month) & $19.9 \pm 3.3$ & $20.5 \pm 3.7$ & 0.916 & 0.362 \\
\hline Ankle dorsiflexion $\left({ }^{\circ}\right)$ & $14.2 \pm 5.0$ & $15.1 \pm 4.8$ & 0.830 & 0.409 \\
\hline Ankle plantarflexion $\left(^{\circ}\right)$ & $25.9 \pm 8.7$ & $27.4 \pm 8.5$ & 0.857 & 0.394 \\
\hline VAS score & $2.9 \pm 1.2$ & $2.6 \pm 0.9$ & 1.239 & 0.218 \\
\hline AOFAS score & $84.7 \pm 9.0$ & $87.4 \pm 8.7$ & 1.456 & 0.149 \\
\hline Excellent $(n)$ & 23 & 24 & 0.273 & 0.602 \\
\hline Good $(n)$ & 14 & 18 & 1.208 & 0.272 \\
\hline Fair $(n)$ & 8 & 2 & 2.454 & $0.117^{\mathrm{a}}$ \\
\hline Poor $(n)$ & 3 & 1 & 0.198 & $0.656^{\mathrm{a}}$ \\
\hline Rate of excellent and good outcome (\%) & $77.1 \%$ & $93.3 \%$ & 4.796 & 0.029 \\
\hline
\end{tabular}

${ }^{\mathrm{a}} P$ value for continuity-corrected chi-squared test.

3D printing group had 5 patients with type $43-\mathrm{C} 1$ fracture, 14 patients with type 43-C2 fracture, and 26 patients with type 43-C3 fracture. Based on the AO soft tissue injury grading, there were 25 patients with grade IC 1,16 patients with grade IC 2, and 7 patients with grade IC 3 in conventional group. And 28 patients with grade IC 1,12 patients with grade IC 2, and 5 patients with grade IC 3 were included in $3 \mathrm{D}$ printing group. The most common cause of injury was fall from a height in both groups (27/48 versus 21/45). There were 38 patients with fibula fractures in conventional group and 33 patients with fibula fracture in $3 \mathrm{D}$ printing group. Time from injury to surgery of conventional group was $8.1 \pm 2.3$ days and it was $7.6 \pm 2.5$ days in $3 \mathrm{D}$ printing group. However, the demographic characteristics such as age, gender, side of injury, fracture classification, soft tissue grading, cause of injury, and time from injury to surgery were similar between the two groups $(P>0.05$; Table 6$)$.

3.2. Clinical Data. The results from clinical data were shown in Table 1 . The operation time in the $3 \mathrm{D}$ printing group was $74.1 \pm 8.2 \mathrm{~min}$, which was significantly shorter than the conventional group $(90.2 \pm 10.9 \mathrm{~min}, P<0.001)$. There was statistical significance in the intraoperative blood loss volume between the $3 \mathrm{D}$ printing group $(117.1 \pm 20.7 \mathrm{ml})$ and the conventional group $(159.8 \pm 26.5 \mathrm{ml}, P<0.001)$. In addition, the $3 \mathrm{D}$ printing group had significantly times of fluoroscopy during the operation $(7.6 \pm 2.2)$ than the conventional group
$(11.0 \pm 2.9, P<0.001)$. Fracture union time was also observed in the patients of the two groups. There was no significant difference in fracture union time between the $3 \mathrm{D}$ printing group $(5.0 \pm 1.1$ months $)$ and the conventional group $(5.3 \pm 1.2$ months, $P=0.314)$. According to Burwell and Charnley grading, 41 patients achieved anatomic reduction, 3 patients achieved fair reduction, and 1 patient achieved poor reduction in the $3 \mathrm{D}$ printing group, while 36 patients achieved anatomic reduction, 8 patients achieved fair reduction, and 4 patients achieved poor reduction in the conventional group. No significant difference was found in fracture reduction between the two groups $(P=0.116)$. However, 3D printing group exhibited significantly higher rate of anatomic reduction (91.1\%) than conventional group (75\%, $P=0.040)$.

3.3. Functional Outcomes. The follow-up of the patients at least was 12 months. There was no statistical significance in the follow-up time between the $3 \mathrm{D}$ printing group $(20.5 \pm 3.7$ months) and the conventional group (19.9 \pm 3.3 months, $P=0.362$ ). Compared to the initial situation, we found the ankle function of the patients in both groups was improved. As shown in Table 2, the motion of ankle dorsiflexion was $15.1 \pm 4.8^{\circ}$ in the $3 \mathrm{D}$ printing group and for the conventional group was $14.2 \pm 5.0^{\circ}$. The motion of ankle plantarflexion was $27.4 \pm 8.5^{\circ}$ in the $3 \mathrm{D}$-printing group and for the conventional group was $25.9 \pm 8.7^{\circ}$. The two groups showed no significant 
TABLE 3: Comparison of complications between the two groups.

\begin{tabular}{|c|c|c|c|c|}
\hline Complications & Conventional group $(n=48)$ & $3 \mathrm{D}$ printing group $(n=45)$ & $\chi^{2}$ & $P$ value \\
\hline Superficial infection & $4(8.3 \%)$ & $3(6.7 \%)$ & - & $1.000^{\mathrm{a}}$ \\
\hline Deep infection & 0 & 0 & - & - \\
\hline Traumatic arthritis & $2(4.2 \%)$ & $1(2.2 \%)$ & - & $1.000^{\mathrm{a}}$ \\
\hline Delayed union & $3(6.3 \%)$ & $2(4.4 \%)$ & - & $1.000^{\mathrm{a}}$ \\
\hline Malunion & $1(2.1 \%)$ & $1(2.2 \%)$ & - & $1.000^{\mathrm{a}}$ \\
\hline Nonunion & 0 & 0 & - & - \\
\hline Total & $10(20.8 \%)$ & $7(15.6 \%)$ & 0.433 & 0.510 \\
\hline
\end{tabular}

Values are expressed as number (\%); ${ }^{\mathrm{a}} \mathrm{P}$ value for Fisher's exact test.

TABLE 4: Questionnaire for doctors.

\begin{tabular}{lcc}
\hline Question & Subjective field & Average scores \\
\hline$(1)$ & Verisimilitude degree of the 3D printing model compared with the actual fracture & $8.5 \pm 1.0$ \\
$(2)$ & Presentation of anatomical structure of fracture & $8.4 \pm 1.2$ \\
$(3)$ & Usefulness of the 3D printing model for diagnosis and preoperative planning & $8.3 \pm 1.1$ \\
$(4)$ & How much dose the 3D printing model help you to communicate with patients? & $8.8 \pm 1.0$ \\
$(5)$ & Overall satisfaction with the 3D printing model & $9.0 \pm 1.1$ \\
\hline
\end{tabular}

TABLE 5: Questionnaire for patients.

Question
$\begin{gathered}\text { How much does the CT or 3D printing model help you to } \\ \text { gain a better communication with doctors? }\end{gathered}$
How much does the CT or 3D printing model help you to
understand the surgical plan?

difference in the range of ankle motion $(P>0.05)$. Besides, the mean VAS score in the $3 \mathrm{D}$ printing group was $2.6 \pm$ 0.9 and for the conventional group was $2.9 \pm 1.2$, which was not statistical different $(P=0.218)$. Furthermore, the mean AOFAS score of the 3D printing group was $87.4 \pm 8.7$, whereas that of the conventional group was $84.7 \pm 9.0$. No significant difference was noted in AOFAS score between the two groups $(P=0.149)$. Moreover, in the $3 \mathrm{D}$ printing group, AOFAS score was scored as excellent in 24 patients, good in 18 patients, fair in 2 patients, and poor in 1 patient. In the conventional group, AOFAS score was scored as excellent in 23 patients, good in 14 patients, fair in 8 patients, and poor in 3 patients. The 3D printing group exhibited a significantly higher rate of good and excellent functional outcome (93.3\%) than the conventional group $(77.1 \%, P=0.029)$.

3.4. Complications. The complications are summarized in Table 3. The total complication rate of the $3 \mathrm{D}$ printing group and the conventional group was $15.6 \%(7 / 45)$ and $20.8 \%(10 / 48)$, which was not statistically different $(P=$ 0.510 ). Three patients with superficial infection were found in the 3D printing group while 4 patients were found in the conventional group, which were all successfully treated with antibiotics and daily dressing. There were 2 patients with delayed union in the 3D printing group while 3 patients were found in the conventional group. However, all of the delayed union patients healed after undergoing reinforcing weight-bearing and functional exercises within 9 months after surgery. Besides, 1 patient in the 3D printing group and 1 patient in the conventional group had radiological evidence of malunion during the follow-up period. One patient in the $3 \mathrm{D}$ printing group and 2 patients in the conventional group had some evidence of posttraumatic arthritis. One patient was managed conservatively and remained under follow-up, with a possibility of requiring surgical intervention in the near future. The other 2 patients had undergone arthroscopic debridement of anterior osteophytes. No other complications such as nonunion and deep infection were found in any group.

3.5. Questionnaire. The questionnaire from doctors showed that the overall satisfaction and usefulness of the $3 \mathrm{D}$ printing models were high (Table 4). For doctors, 3D printing models could provide a visual, comprehensive vision of fracture displacement, which can make the preoperative plan much easier. On the other hand, patients and their family members gave relatively high satisfaction scores for the quality of preoperative communication when the $3 \mathrm{D}$ printed fracture model was introduced to help the surgeons explain the patients' medical condition (Table 5 and Figure 6).

3.6. Typical Case. Male, 52 years old, falling from height, was selected as a typical case. Figure 2 showed the preoperative Xray and CT scan of fracture. Using the CT bone segmentation and mirror imaging technique in Mimics software v17.0, the characteristics of the injured tibia and the tibia before the injury were exhibited clearly (Figure 3). Afterwards, a $1: 1$ solid prototype of the fracture and the mirror model of normal side tibia were manufactured by $3 \mathrm{D}$ printing 


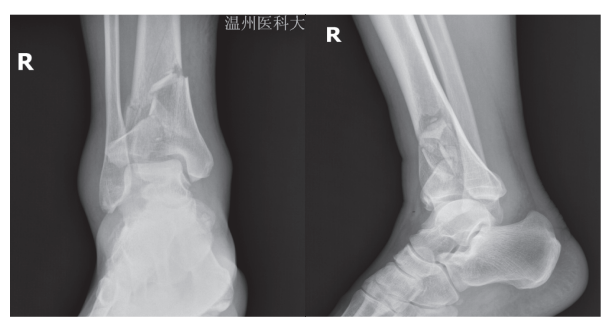

(a)

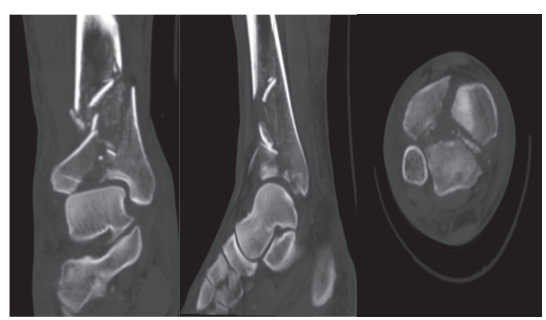

(b)

Figure 2: The patient's preoperative radiological characteristics of Pilon fracture. (a) The anteroposterior and lateral X-ray. (b) CT images of the fracture.

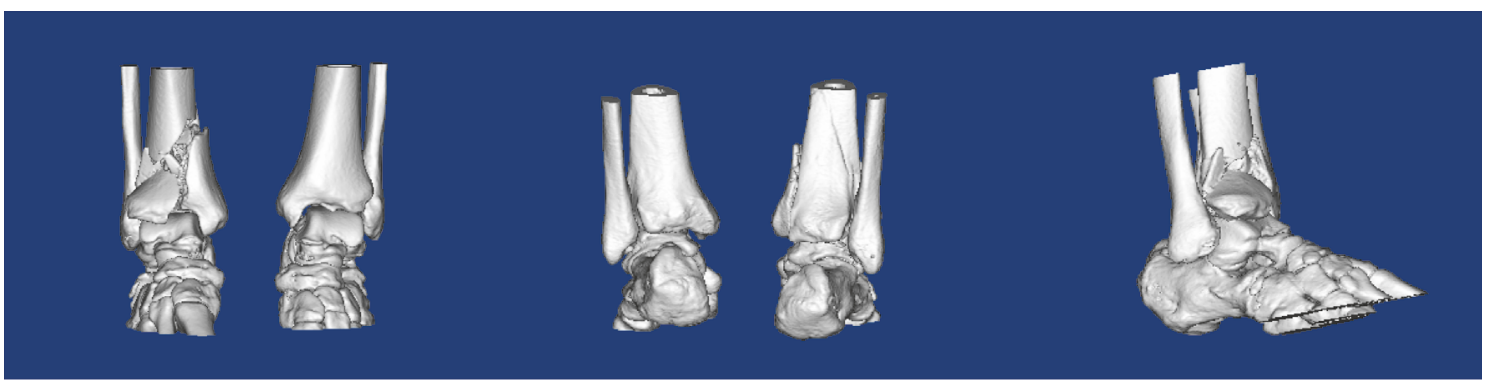

(a)

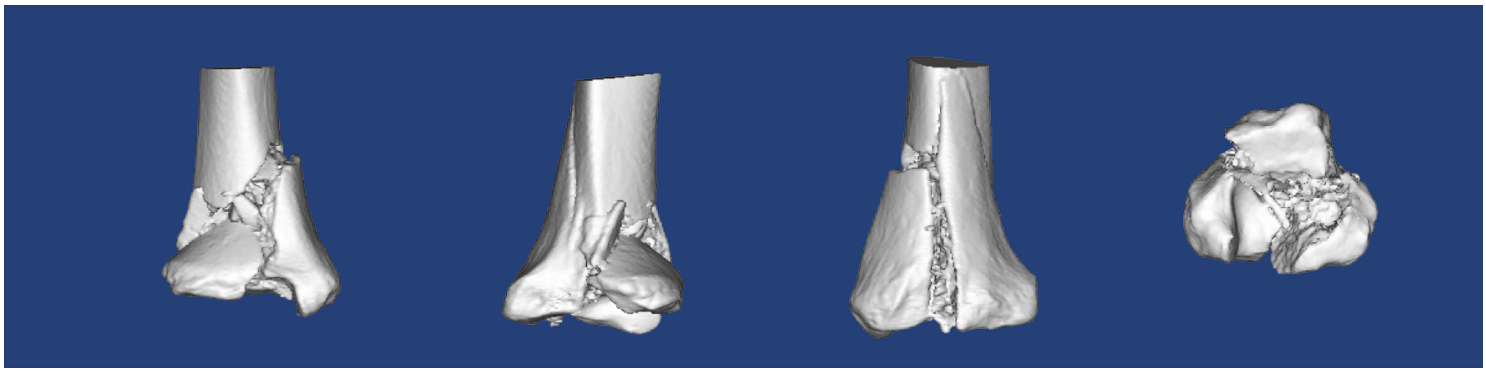

(b)

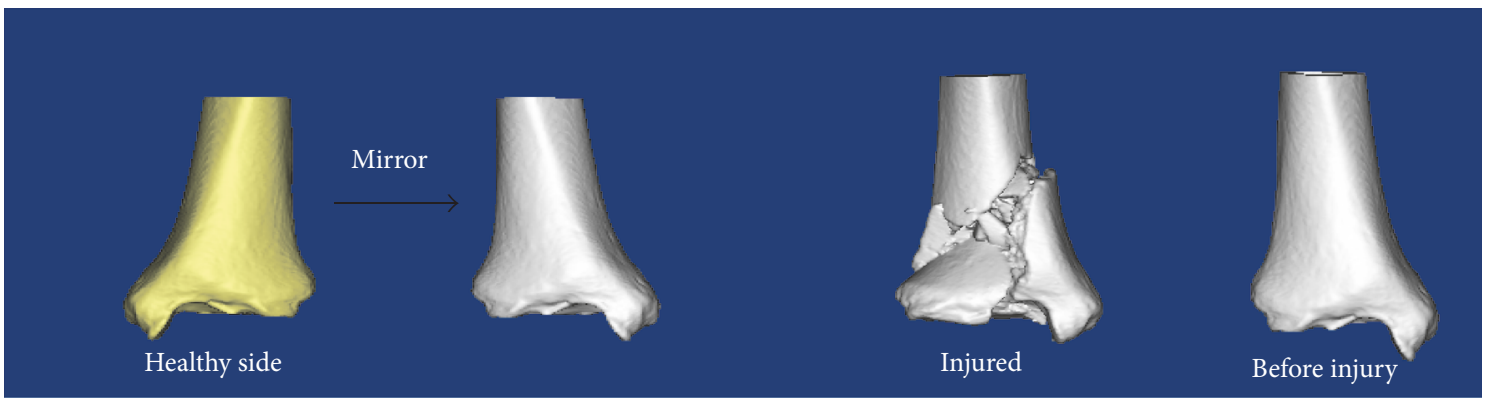

(c)

FIgURE 3: The 3D reconstruction and manipulation of the fracture in Mimics software v17.0. (a) The 3D reconstruction of Pilon fracture. (b) Using the CT bone segmentation function to separate the tibia. (c) Using the mirror imaging technique to reconstruct the model of tibia before injury.

technology; then surgeons simulated the operation on the real-size mirror normal 3D printed tibia model. The outcome of the simulative operation was shown in Figure 4. Then the simulative operation was used to guide the actual operation. Postoperative review of X-ray film showed satisfactory fracture reduction and fixation (Figure 5). The plate and screw were in good position. This patient had a followup of 21 months. In this period, he did not suffer from any surgical complications and exhibited excellent AOFAS score. 


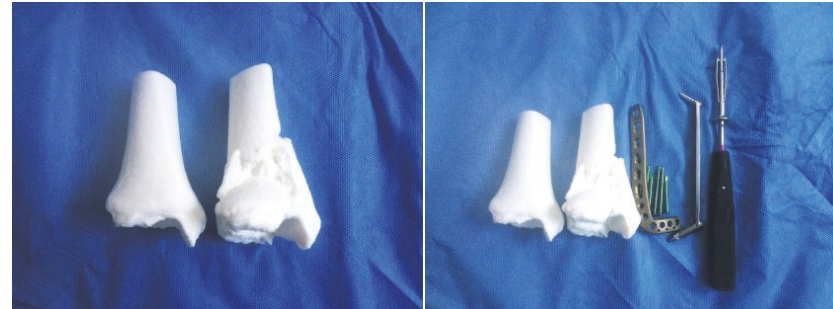

(a)

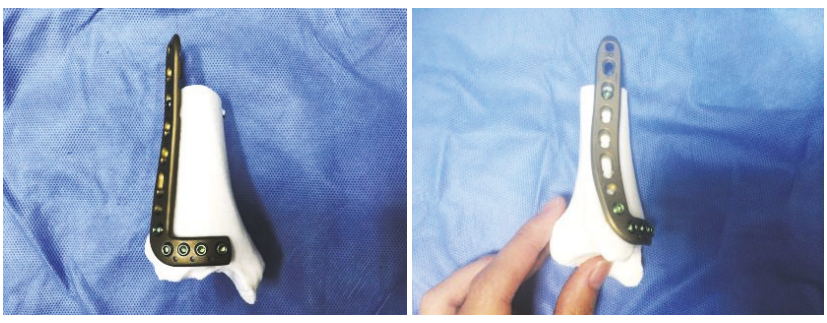

(c)
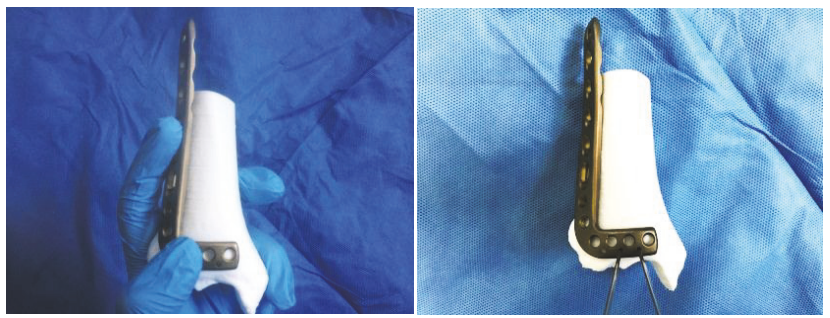

(b)
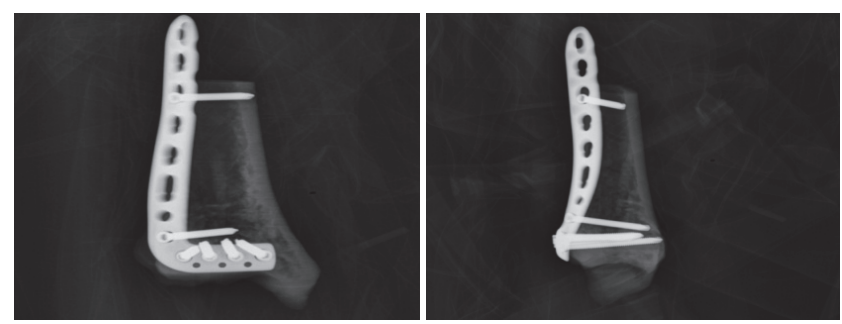

(d)

Figure 4: Simulation of the surgery in vitro. (a) Preparation of simulative surgery. (b-c) Simulating the surgery on the model. (d) The anteroposterior and lateral X-ray after the simulative surgery.

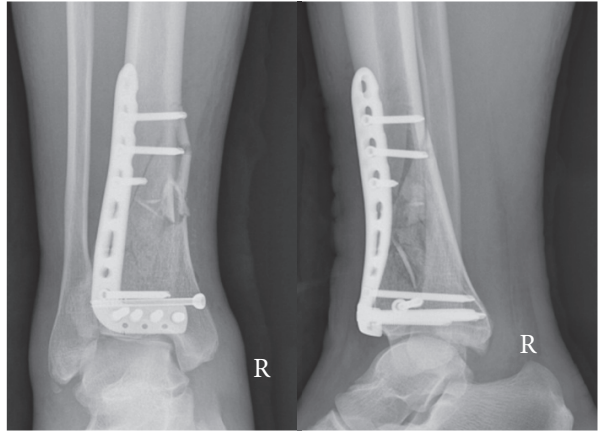

(a)

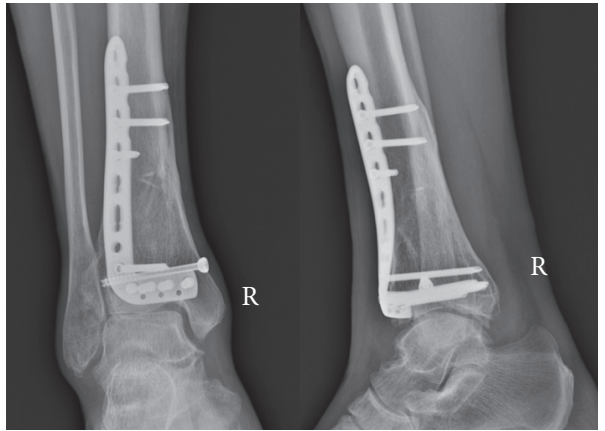

(c)

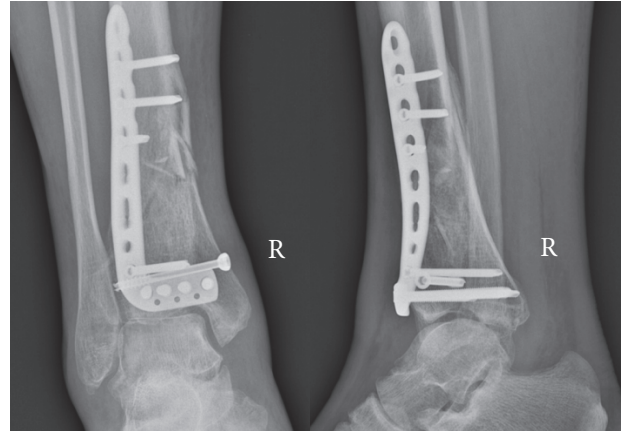

(b)

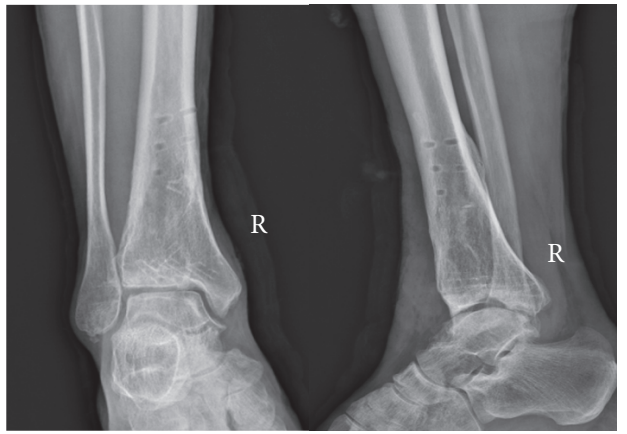

(d)

FIGURE 5: The radiography after the operation. (a) The anteroposterior and lateral X-ray after operation. (b) The anteroposterior and lateral $\mathrm{X}$-ray at 3 months postoperatively. (c) The anteroposterior and lateral X-ray at 6 months postoperatively. (d) The anteroposterior and lateral $\mathrm{X}$-ray at 18 months postoperatively (after internal fixation removal).

\section{Discussion}

It is well known that the main goal of surgical treatment for Pilon fractures is to anatomically reduce and fix the intraarticular fragments and restore the length, alignment, and rotation of the distal tibia, which allows for earlier weightbearing and mobilization $[3,17]$. Therefore, a better overall preoperative understanding of the anatomical structure of the fracture is quite essential for the treatment. However, the $\mathrm{X}$-ray and CT images could not provide a comprehensively 
TABLE 6: Comparison of the general conditions of the two groups.

\begin{tabular}{|c|c|c|c|c|}
\hline & Conventional group & $3 \mathrm{D}$ printing group & $t$ or $\chi^{2}$ & $P$ value \\
\hline$n$ & 48 & 45 & & \\
\hline \multicolumn{5}{|l|}{ Gender } \\
\hline Male & 31 & 35 & \multirow{2}{*}{1.963} & \multirow{2}{*}{0.161} \\
\hline Female & 17 & 10 & & \\
\hline Age (year) & $42.5 \pm 9.0$ & $41.2 \pm 9.3$ & 0.698 & 0.487 \\
\hline \multicolumn{5}{|l|}{ Cause of injury } \\
\hline Fall from a height & 27 & 21 & \multirow{3}{*}{0.856} & \multirow{3}{*}{0.652} \\
\hline Traffic accident & 15 & 17 & & \\
\hline Other causes & 6 & 7 & & \\
\hline \multicolumn{5}{|l|}{ Side of injury } \\
\hline Left & 20 & 23 & \multirow{2}{*}{0.833} & \multirow{2}{*}{0.361} \\
\hline Right & 28 & 22 & & \\
\hline \multicolumn{5}{|l|}{ AO/OTA classification } \\
\hline $43-\mathrm{Cl}$ & 8 & 5 & \multirow{3}{*}{1.071} & \multirow{3}{*}{0.585} \\
\hline $43-\mathrm{C} 2$ & 17 & 14 & & \\
\hline $43-\mathrm{C} 3$ & 23 & 26 & & \\
\hline \multicolumn{5}{|l|}{ AO soft tissue grading } \\
\hline IC 1 & 25 & 28 & \multirow{3}{*}{0.979} & \multirow{3}{*}{0.613} \\
\hline IC 2 & 16 & 12 & & \\
\hline IC 3 & 7 & 5 & & \\
\hline Associated fibula fracture $(n)$ & 38 & 33 & 0.438 & 0.508 \\
\hline Time from injury to operation (day) & $8.1 \pm 2.3$ & $7.6 \pm 2.5$ & 1.062 & 0.291 \\
\hline
\end{tabular}

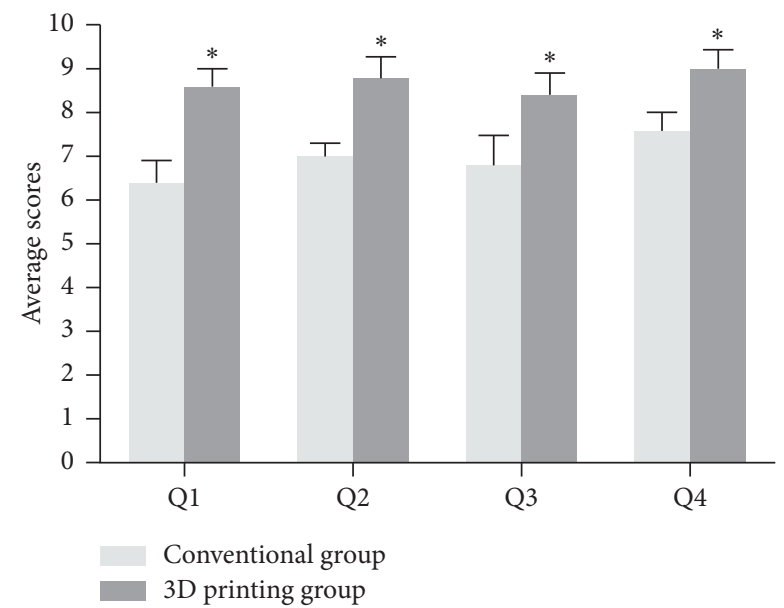

FIGURE 6: Survey questionnaire for patients and nonmedical professionals between the two groups. $* \mathrm{P}<0.05$ compared with conventional group.

understanding for the fracture, no more for the private custom.

In recent years, the application of the $3 \mathrm{D}$ printing applied in orthopedics was more and more common [18-20]. In this study, we found that $3 \mathrm{D}$ skeletal models can be printed according to patient CT data in combination with modern digital medical technology. Using 3D printed model, the fracture can be viewed in every direction to provide an accurate description of fracture characteristics. This enables the orthopedist to more effectively and comprehensively understand the specific details of the fracture, confirm the type of fracture, determine the displacement of the fracture line and the number of fracture fragments, examine the collapse and comminuting condition of the articular surface, verify the potential presence of bone defects, and determine whether bone graft is needed. Besides, $3 \mathrm{D}$ printing model is able to help orthopedist to make an individual, accurate, and reasonable surgical plan for patients. Surgeon can clearly and directly visualize the anatomical and pathological conditions of the fracture sites before surgery rather than having to begin operating with only partial knowledge. Therefore, the surgeon can accurately make surgical plans and simulate the surgical procedures before the procedure, which can highly increase the accuracy of reduction and the stability of the fixation [21]. The 3D printing also was used in ankle fractures and clavicular fractures, which showed its unique advantages, reducing the operation time, intraoperative blood loss, and the plasticity of plate $[22,23]$.

In the present study, we compared the conventional surgery with the surgery assisted by $3 \mathrm{D}$ printing technology in the treatment of Pilon fractures. In 3D printing group, we used the mirror technology to assist preoperative reduction, making the preoperative design, and simulate the operation. The size and location of the plate and screw could be made out. So it may account for the reduced operation time in the $3 \mathrm{D}$ printing group and may additionally help reduce intraoperative bleeding and fluoroscopy times leading to better functional recovery. Our results showed that the $3 \mathrm{D}$ printing group exhibited significantly shorter operation time, 
less intraoperative blood loss volume, and less times of intraoperative fluoroscopy as well as higher rate of anatomic reduction than the conventional group, suggesting that the suitable preoperative planning and individual treatment determined by $3 \mathrm{D}$ printed model could greatly optimize the surgical outcomes. In addition, this can reduce the exposure to radiation both for patients and for doctors and reduce the potential injury from surgery and anesthesia. However, the two groups did not differ significantly in functional outcome at the last follow-up period. As for the postoperative complication, there were no significant differences between 3D printing group and conventional group (15.6\% and 20.8\%). There is no obvious advantage of $3 \mathrm{D}$ printing technology for the prevention of complications.

The doctor-patient communication is very important in the clinic process [24]. It has been reported that $3 \mathrm{D}$ printed models could improve patient's understanding and compliance in orthopedic surgery $[25,26]$. Fully understanding the fracture conditions can help patients and their family members coordinate surgical treatment with functional rehabilitation after surgery. In the current study, we made a questionnaire for both doctors and patients. According to Figure 6, conventional medical images are too complicated for patients and nonmedical professionals to fully understand, which increased the difficulty of doctor-patient communication. Our study found that the overall patient evaluation of the quality of doctor-patient communication was above 9 out of 10 points when the 3D fracture model was introduced to help explain the medical conditions and surgical plan to patients and their family members. Patients and their family members were thus satisfied with this type of communication, which effectively increased the ability of the patient or family member to understand the patient's medical condition while improving patient attitude toward and compliance with the doctor's recommendations [27]. On the other hand, doctors can also gain a better communication with both patients and their working team, which not only increases patient's understanding and compliance but also improves the performance and the collaboration of working team. Therefore, 3D printed model may be an innovative tool to improve the doctor-patient communication and achieve better clinical outcomes of the fractures.

Nonetheless, there are still some limitations for the 3D printing. Firstly, the 3D printing technology used in our study is only based on bone CT images which lack the information of the adjoining soft tissue and vasculature. Moreover, it will take a lot of preparation time for the model printing and simulated surgery; therefore it is not suitable for emergency cases. Also, 3D printing technology needs specific software, professionals, and 3D printers, which will undoubtedly increase human and financial expenses. Besides, this study enrolled only a small number of patients. To further confirm these results, high-quality randomized controlled trials with larger sample size were still needed.

Finally, in our study, 3D printing technology is still in the scientific research stage, not for charging, and our research funding supports it. What is more, the most work was done by our master graduate students. If this technology is promoted, more health resources will be needed.

\section{Conclusion}

In conclusion, 3D printing technology has provided orthopedic surgeons with powerful new tools and approaches. This technology is both safe and effective for the treatment of adults with Pilon fractures and has a significantly shorter operative time, less intraoperative blood loss, less times of fluoroscopy, and higher rate of anatomic reduction compared with the conventional group. Furthermore, 3D printing can help clinicians improve their theoretical knowledge and practical skills, reduce learning curves, eliminate common surgical complications, improve surgical quality, and provide a better communication between doctors and patients.

\section{Ethical Approval}

The study was approved by the Ethical Board Review of our hospital and was performed in accordance with the ethical standards of the Declaration of Helsinki of 1964.

\section{Conflicts of Interest}

The authors declare that there are no conflicts of interest regarding the publication of this article.

\section{Acknowledgments}

The authors thank all the staff in the Laboratory of Orthopaedic Research Institute and Scientific Research Center of the Second Affiliated Hospital and Yuying Children's Hospital of Wenzhou Medical University. This study was funded by the National Nature Foundation of China (no. 81701928), Zhejiang Province Medical and Health Technology Project (2017KY480), and Zhejiang Province College Students Science and Technology Innovation Program (no. 2017R413079).

\section{References}

[1] S. Vidyadhara and S. K. Rao, "Ilizarov treatment of complex tibial pilon fractures," International Orthopaedics, vol. 30, no. 2, pp. 113-117, 2006.

[2] S. Bacon, W. R. Smith, S. J. Morgan et al., "A retrospective analysis of comminuted intra-articular fractures of the tibial plafond: Open reduction and internal fixation versus external Ilizarov fixation," Injury, vol. 39, no. 2, pp. 196-202, 2008.

[3] A. D'Heurle, N. Kazemi, C. Connelly, J. D. Wyrick, M. T. Archdeacon, and T. T. Le, "Prospective randomized comparison of locked plates versus nonlocked plates for the treatment of high-energy pilon fractures," Journal of Orthopaedic Trauma, vol. 29, no. 9, pp. 420-423, 2015.

[4] J. Conroy, M. Agarwal, P. V. Giannoudis, and S. J. E. Matthews, "Early internal fixation and soft tissue cover of severe open tibial pilon fractures," International Orthopaedics, vol. 27, no. 6, pp. 343-347, 2003.

[5] D. J. Hak, M. Lee, and D. R. Gotham, "Influence of prior fasciotomy on infection after open reduction and internal fixation of tibial plateau fractures," Journal of Trauma - Injury Infection and Critical Care, vol. 69, no. 4, pp. 886-888, 2010. 
[6] M. A. McFerran, S. W. Smith, H. J. Boulas, and H. S. Schwartz, "Complications encountered in the treatment of pilon fractures," Journal of Orthopaedic Trauma, vol. 6, no. 2, pp. 195-200, 1992.

[7] L. Dillin and P. Slabaugh, "Delayed wound healing, infection, and nonunion following open reduction and internal fixation of tibial plafond fractures," Journal of Trauma - Injury Infection and Critical Care, vol. 26, no. 12, pp. 1116-1119, 1986.

[8] M. Azuma, T. Yanagawa, N. Ishibashi-Kanno et al., "Mandibular reconstruction using plates prebent to fit rapid prototyping 3dimensional printing models ameliorates contour deformity," Head \& face medicine, vol. 10, p. 45, 2014.

[9] H.-S. Jeong, K.-J. Park, K.-M. Kil et al., "Minimally invasive plate osteosynthesis using 3D Printing for shaft fractures of clavicles: technical note," Archives of Orthopaedic and Trauma Surgery, vol. 134, no. 11, pp. 1551-1555, 2014.

[10] C. Li, M. Yang, Y. Xie et al., "Application of the polystyrene model made by 3-D printing rapid prototyping technology for operation planning in revision lumbar discectomy," Journal of Orthopaedic Science, vol. 20, no. 3, article no. 706, pp. 475-480, 2015.

[11] X. Tang, L. Liu, C.-Q. Tu, J. Li, Q. Li, and F.-X. Pei, “Comparison of early and delayed open reduction and internal fixation for treating closed tibial pilon fractures," Foot \& Ankle International, vol. 35, no. 7, pp. 657-664, 2014.

[12] T. Ibrahim, A. Beiri, M. Azzabi, A. J. Best, G. J. Taylor, and D. K. Menon, "Reliability and validity of the subjective component of the american orthopaedic foot and ankle society clinical rating scales," Journal of Foot and Ankle Surgery, vol. 46, no. 2, pp. 6574, 2007.

[13] H. N. Burwell and A. D. Charnley, "The treatment of displaced fractures at the ankle by rigid internal fixation and early joint movement," The Journal of Bone \& Joint Surgery (British Volume), vol. 47, no. 4, pp. 634-660, 1965.

[14] M. Ronga, U. G. Longo, and N. Maffulli, "Minimally invasive locked plating of distal tibia fractures is safe and effective," Clinical Orthopaedics and Related Research, vol. 468, no. 4, pp. 975-982, 2010.

[15] A. Nehme, Z. Tannous, J. Wehbe, R. Mecharrafieh, and G. Maalouf, "Arthroscopically Assisted Reconstruction and Percutaneous Screw Fixation of a Pilon Tibial Malunion," Journal of Foot and Ankle Surgery, vol. 46, no. 6, pp. 502-507, 2007.

[16] M. F. Swiontkowski, A. K. Sands, J. Agel, M. Diab, J. R. Schwappach, and H. J. Kreder, "Interobserver variation in the AO/OTA fracture classification system for pilon fractures: Is there a problem?" Journal of Orthopaedic Trauma, vol. 11, no. 7, pp. 467-470, 1997.

[17] A. Lomax, A. Singh, N. Jane Madeley, and C. Senthil Kumar, "Complications and early results after operative fixation of 68 pilon fractures of the distal tibia," Scottish Medical Journal, vol. 60, no. 2, pp. 79-84, 2015.

[18] P. Benum, A. Aamodt, and L. Nordsletten, "Customised femoral stems in osteopetrosis and the development of a guiding system for the preparation of an intramedullary cavity: A report of two cases," The Journal of Bone \& Joint Surgery (British Volume), vol. 92, no. 9, pp. 1303-1305, 2010.

[19] R. I. Davidovitch, Y. Weil, R. Karia et al., "Intraoperative syndesmotic reduction: three-dimensional versus standard fluoroscopic imaging," The Journal of Bone \& Joint Surgery-American Volume, vol. 95, no. 20, pp. 1838-1843, 2013.

[20] F. Chana-Rodriguez, R. P. Mananes, J. Rojo-Manaute, P. Gil, J. M. Martínez-Gómiz, and J. Vaquero-Martín, "3D surgical printing and pre contoured plates for acetabular fractures," Injury, vol. 47, no. 11, pp. 2507-2511, 2016.

[21] Y. Zhuang, S. Cao, Y. Lin, R. Li, G. Wang, and Y. Wang, "Minimally invasive plate osteosynthesis of acetabular anterior column fractures using the two-incision minimally invasive approach and a preshaped three dimension plate," International Orthopaedics, vol. 40, no. 10, pp. 2157-2162, 2016.

[22] L. Yang, X.-W. Shang, J.-N. Fan et al., "Application of 3D Printing in the Surgical Planning of Trimalleolar Fracture and DoctorPatient Communication," BioMed Research International, vol. 2016, Article ID 2482086, 2016.

[23] N. H. Kim, N. X. Liu, and C. K. Noh, "Use of a real-size 3Dprinted model as a preoperative and intraoperative tool for minimally invasive plating of comminuted midshaft clavicle fractures," Journal of Orthopaedic Surgery and Research, vol. 10, no. 1, article no. 91, 2015.

[24] L. Dyche, "Interpersonal skill in medicine: The essential partner of verbal communication," Journal of General Internal Medicine, vol. 22, no. 7, pp. 1035-1039, 2007.

[25] Y. Lou, L. Cai, C. Wang et al., "Comparison of traditional surgery and surgery assisted by three dimensional printing technology in the treatment of tibial plateau fractures," International Orthopaedics, vol. 41, no. 9, pp. 1875-1880, 2017.

[26] W. Zheng, Z. Tao, Y. Lou et al., "Comparison of the Conventional Surgery and the Surgery Assisted by 3d Printing Technology in the Treatment of Calcaneal Fractures," Journal of Investigative Surgery, pp. 1-11, 2017.

[27] S.-H. Won, Y.-K. Lee, Y.-C. Ha, Y.-S. Suh, and K.-H. Koo, "Improving pre-operative planning for complex total hip replacement with a Rapid Prototype model enabling surgical simulation," The Bone \& Joint Journal, vol. 95, no. 11, pp. 14581463, 2013. 


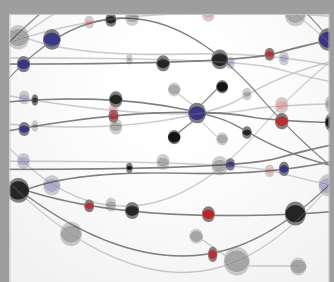

The Scientific World Journal
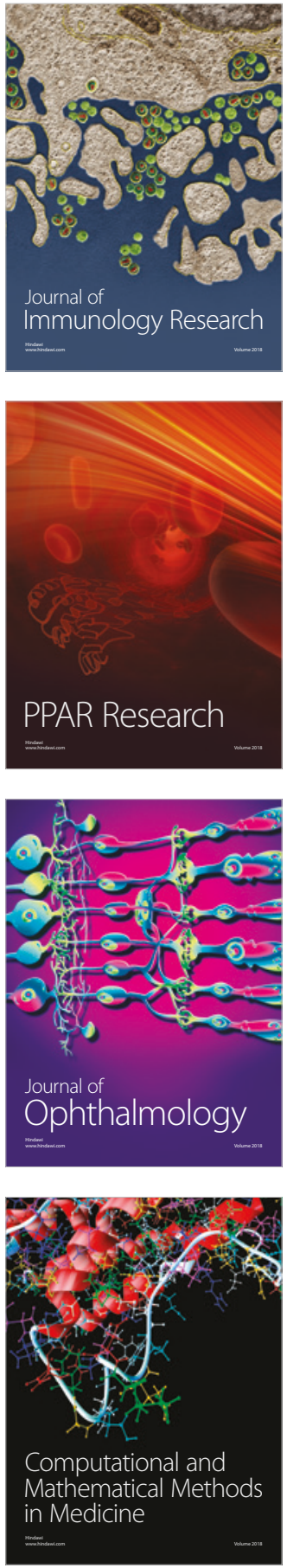

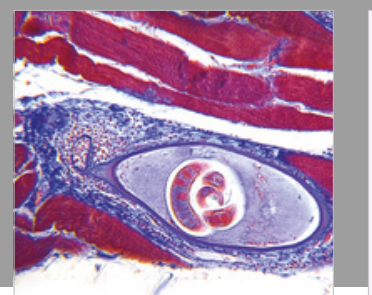

Gastroenterology Research and Practice

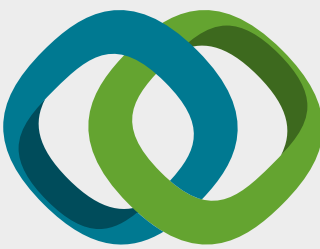

\section{Hindawi}

Submit your manuscripts at

www.hindawi.com
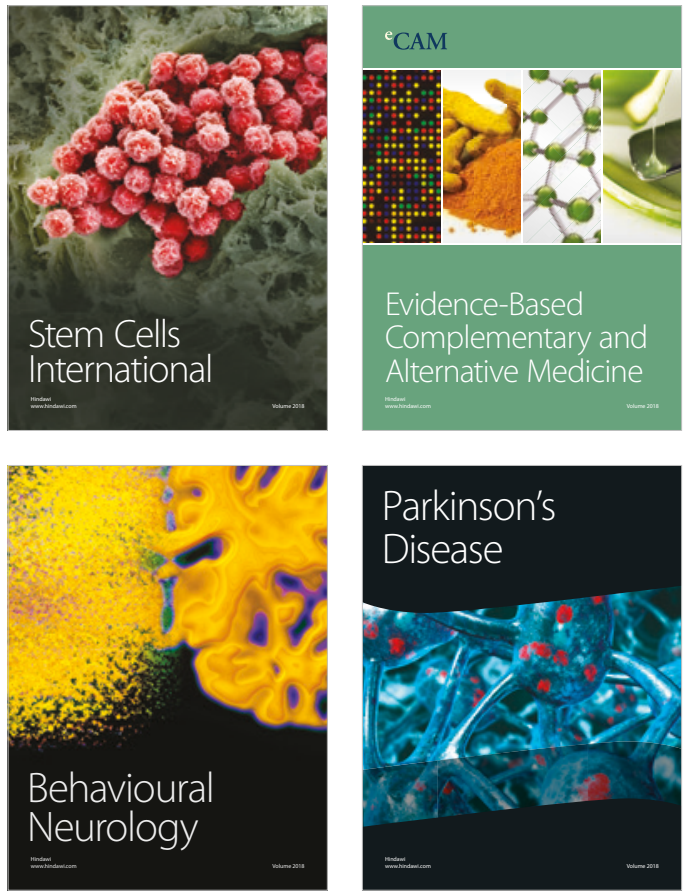

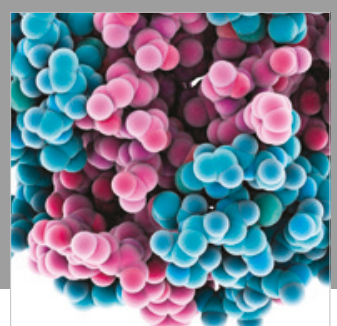

ournal of

Diabetes Research

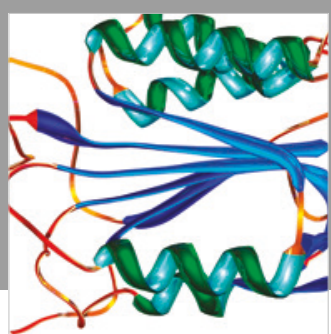

Disease Markers
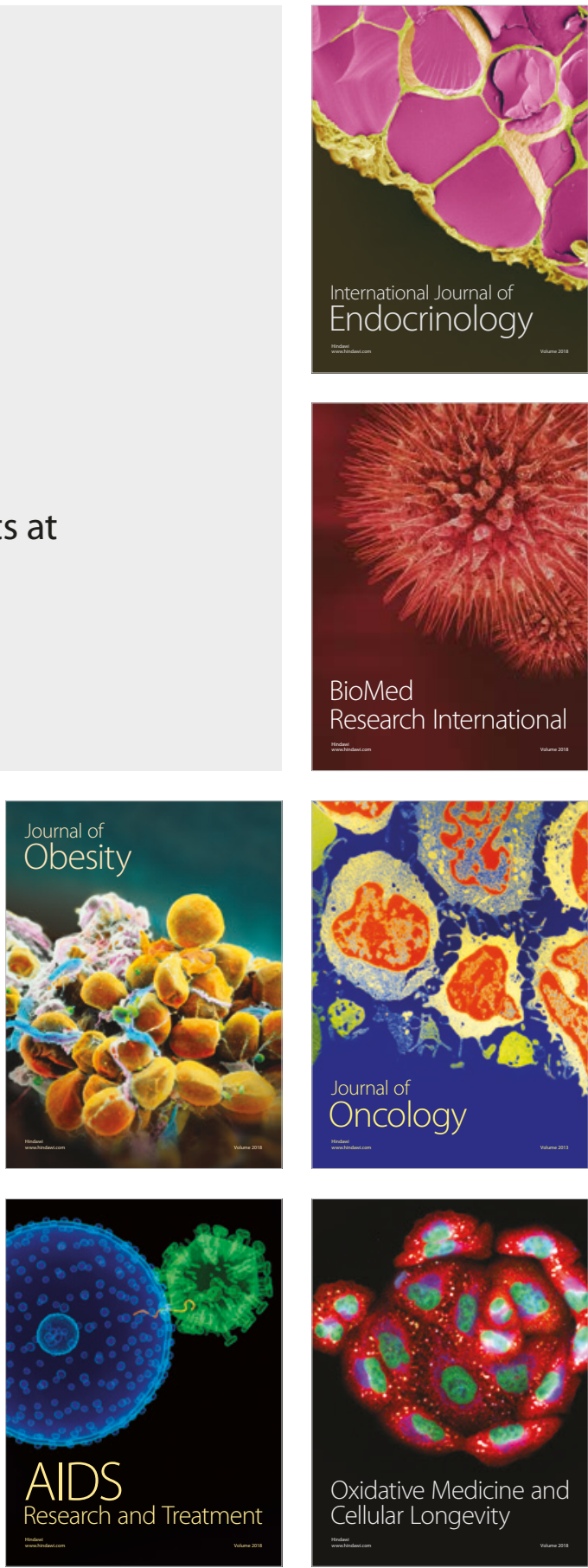